\title{
Implementation of JSON Update Framework in RDBMSs
}

\author{
Dušan Petković \\ University of Applied Sciences \\ Hochschulstr. 1, Rosenheim, 83024, Germany
}

\begin{abstract}
The existing SQL/JSON standard specifies the way how queries can be performed, but in order to reach the full functionality for data representation and as a sharing format, it needs to support several additional features. One of the most important missing features is the facility to perform updates on the content of JSON documents. In this article we discuss how update operations for JSON data have been implemented in relational database systems and compare these implementations in relation to their scope and coverage of our framework, which comprises the set of primitive update operations.
\end{abstract}

SQL Server uniformly supports all implemented operations with the JSON_MODIFY function, but the syntax of the second argument of the function is not uniform. The syntax for PostgreSQL update operations is rather awkward and the implementation is not uniform. On the other hand, PostgreSQL supports the full update operation in the same way as this paper proposes. MySQL implemented all update primitives specified in the framework.

\section{Keywords}

JSON, SQL/JSON, JSON Update Framework, MySQL, PostgreSQL, SQL Server

\section{INTRODUCTION}

The role of JSON (JavaScript Object Notation) in the last couple of years has expanded significantly. The reason for this expansion is that JSON is a data format that can represent structured as well as semi-structured data in a simple way. The structure of JSON content follows the syntax structure for JavaScript. The following example:

info: $\{\{$ "who": "Fred", "where": "BBC",

"friends":

[\{"name":"Lili","rank":5\},\{"name":"Hank","rank": 7\}]\}\}

shows a JSON document called info that describes a single person, Fred, his affiliation, BBC, and his friends, Lili and Hank. Generally, a JSON string contains either an array of values or an object, which is an array of name/value pairs. (The description of the JSON data model is given below.)

\subsection{Data Model}

A JSON text is a sequence of tokens formed from Unicode code points that conforms to the JSON value grammar. A JSON value can be an object, array, number, string, true, false, or null. An object is represented as a pair of curly bracket tokens surrounding zero or more name/value pairs. A name is a string. A single colon token follows each name, separating the name from the value. An array is a pair of square bracket tokens surrounding zero or more values. The values are separated by commas. The JSON syntax does not define any specific meaning to the ordering of the JSON values. However, the JSON array structure is used in situations where there is some semantics to the ordering. A number is a sequence of decimal digits. It may have a preceding minus sign and/or a fractional part prefixed by a decimal point. A string is a sequence of Unicode code points wrapped with quotation marks.

\subsection{The SQL/JSON Standard}

The SQL/JSON standard has been first published as "change proposal" in March, 2014 in two documents. The first part of the change proposal [14] provides an introduction to JSON and discusses SQL operators, which can be used to construct JSON documents stored as character or binary strings. The second part specifies how JSON can be queried in relational environment [15].

The revised version of the SQL standard with JSON support includes some additional corrections and was officially published in December, 2016. The corresponding "Technical Report", which was published in March, 2017 can be downloaded for free [2].

As discussed in [8], the existing specification of the standard is "light-weighted", meaning that JSON objects cannot be stored in a database system in a native form. One of the reasons for this decision is to allow vendors of relational database systems (RDBMS) to implement the proposed features in their systems as soon as possible.

Two very important missing features in the SQL/JSON standard are how the context of JSON documents can be updated and how full-text search can be performed. The current SQL/JSON standard specification can express powerful structural queries over JSON documents, but the specified functions and predicates can be used only to read the existing content. In other words, it should be possible to modify content of these documents and it is exactly the issue of this paper: to specify how the Update JSON functionality, as a part of the SQL/JSON specification, should look like.

The existing SQL/JSON standard lacks the support for queries concerning full-text search, too. In the article [10], the authors propose a set of design goals that full-text search extension of the SQL/JSON language should support. They also discuss fulltext language extensions implemented in relational database systems concerning JSON documents and answer the question to what extent the extensions are supported by these systems.

This paper is structured as follows. Section 2 describes the framework with all update primitives, which should be specified for JSON Update. It handles the necessary operations for insertion, deletion and replacement of the specified content in a JSON document. In three subsections of Section 3, the implementation of update primitives is discussed in relation to three RDBMSs (SQL Server, PostgreSQL and MySQL, respectively). Finally, Section 4 summarizes the results and compares the given framework with the existing implementations.

\subsection{Related Work}

To the best of the author's knowledge, there is no article discussing the framework and the implementation of JSON Update in relational environment. The sketchy discussion of the 
JSON_UPDATE function in relation to the future specification in the SQL/JSON is given in [4]. The article [5] discusses the performance of JSON update operations in general terms. It opts for delta-update operations of a JSON document, which involves structural changes, instead of just leaf value ones. (This is opposite to the current practice, which updates the full JSON instance physically, even though logically only small parts of it need to be updated.)

There is an article concerning XML Update [13]. Although XML as a data format differs significantly from JSON, both formats are based upon hierarchy of data. For this reason, the referenced article gives helpful hints in relation to JSON Update.

\section{UPDATE PRIMITIVES}

The following update primitives should be implemented for each JSON document in a relational environment. The primitives will be separately discussed in relation to the three operations: insertion, deletion and replacement. These update primitives build the framework for JSON update operations.

The insert operation inserts a new content in the specified JSON document. The following cases exist:

a) Insert an object

b) Insert an array member after another array member (new array member must be of the same data type as the existing members of the array)

c) Insert an array member before another array member

(Note that the new array member must be of the same data type as the existing members of the array)

d) Insert an object after another one

(Note that the operation "inserting an object after another one" is optional, because there is no ordering of objects, generally.)

e) Inserts an object before another one

(Note that the operation "inserting an object before another one" is optional, because there is no ordering of objects)

The delete operation deletes the content of the specified JSON document under the given path expression. The following cases exist:

a) Delete the n-th element of the array with the given name

b) Delete an object with the given name

The replace operation replaces the content under the given path expression. The following cases exist:

a) Replace the value of the object with the given name

b) Replace the $n$-th element of the array with the given name

A full update operation may consist of several of the primitive operations, which are executed in sequence and using the comma sign to combine several primitive operations. Therefore, two additional restrictions have been added to the semantics of the operations in order to prevent ill-defined semantics:

a) All bindings within the sequence of primitive operations are made over the input inside a transaction. b) Content is evaluated for each target before the sequence of updates is executed.

\section{UPDATING JSON IN RD BMSs}

Until now, three relational DBMSs have implemented update operations for JSON documents: MS SQL Server, PostgreSQL and MySQL. In this section we will show the semantics and the syntax of these implementations. The general discussion how RDBMSs should integrate JSON documents is given in $[1,3]$. The overview of JSON features already implemented in relational systems is given in [9].

\subsection{SQL Server}

Since SQL Server 2016, this RDBMS supports the JSON_MODIFY function as a part of the SQL UPDATE statement to update parts of a JSON document stored in the column of the NVARCHAR data type. (The general discussion about the integration of JSON in SQL Server can be found in [6], while the description of the JSON_MODIFY function is given in [7].) The following examples show operations supported by SQL Server.

Example 1

-- Create a table with the column which contains JSON documents

CREATE TABLE json_update_table

(id INT PRIMARY KEY IDENTITY, person_and friends NVARCHAR(2000));

-- Insert a row

INSERT INTO json_update_table (person_and_friends) VALUES

(N'\{"info":\{"who": "Fred" , "friends":["Lili", "Hank"]\}\}');

Example 2

-- Insert a new object with a key/value pair: "surname"/"Birch"

UPDATE json_update_table

SET person_and_friends $=$

JSON_MODIFY(person_and_friends, '\$.info.surname', 'Birch')

WHERE id $=1$;

To insert a new element at the end of an array with the given name, use the append option of the JSON_MODIFY function. (The syntax of the second argument of the JSON_MODIFY function is generally of the form "path_expression, value". The append option is the only exception and is used as the prefix of the second argument.)

Example 3

-- Append the element to an array

UPDATE json_update_table

SET person_and_friends=

JSON_MODIFY(person_and_friends, 'append \$.info.friends', 'Wendy')

WHERE id $=1$;

The insertion of an array member before (after) another one cannot be done directly. The way, how this can be done indirectly is given in the following example.

xample 4

-- Insert an array element using DELETE and INSERT 
UPDATE json_update_table

SET person_and_friends =

JSON_MODIFY(person_and_friends, '\$.info.friends', 'Peter', 'Lili', 'Hank', 'Wendy')

WHERE id $=1$;

The JSON_MODIFY function deletes an object with the given key using the NULL value, as shown in the following example. The requirement is that the specified mode is lax. (The implementation of JSON in SQL Server supports two standardized modes, strict and lax [1].)

Example 5

-- Delete an object

UPDATE json_update_table

SET person_and_friends

JSON_MODIFY(person_and_friends, '\$.info.surname', NULL)

WHERE id $=1$;

Example 6

-- Replace the value of an object with the given name

UPDATE json_update_table

SET person_and_friends

JSON_MODIFY(person_and_friends, '\$.info.who', 'Peter')

WHERE id $=1$;

The JSON_MODIFY function updates only one property at time. To execute multiple updates, the JSON_MODIFY function must be invoked inside another JSON_FUNCTION, as shown in the following example. (This corresponds to the requirement in Section 2 that a full update operation may consist of several primitive operations.)

Example 7

-- Multiple updates

DECLARE @info

NVARCHAR(100)='\{ "name":"John","skills":["C\#","SQL"]\}'

SET @info=JSON_MODIFY

(JSON_MODIFY

(JSON_MODIFY(@info,'\$.name','Mike'),'\$.surname','Smith'), 'append \$.skills','Azure')

\subsection{PostgreSQL}

PostgreSQL supports the jsonb_set function as a part of the SQL UPDATE statement to update parts of a JSON document stored in the column of the jsonb data type. This function is available since Version 9.5. The JSON data types are described in [11], while the jsonb_set function is described, together with other JSON functions in [12]. The following statements show JSON update basic operations, supported by this RDBMS.

Example 8

-- Create table

CREATE TABLE json_table(id serial, data jsonb);

-- Insert a row

INSERT INTO json_table(data) values

('\{ "name": "Fred", "friends": ["Bob", "Jane"]\}');
The way, how PostgreSQL supports insertion of a new element of an array is not straightforward, although the syntax seems to be so. The UPDATE statement below inserts a new element of the array using DELETE and INSERT.

Example 9

-- Insert a new element of the array

UPDATE json_table SET data $=$ jsonb_set $($ data, ' $\{$ friends $\}$ ', '["Bob", "Maria", "Jane"]');

The following statement deletes an object with the given name, using rather awkward syntax to specify that primitive update operation.

Example 10

-- Delete an object with the given name

UPDATE json_table SET data = data \#- ' $\{$ friends, -1$\}$ '

The following example appends a new value to the array with the given name, using very large index (999) as the second argument, besides the array name.

Example 11

-- Append an element of an array with the given name

UPDATE json_table SET data = jsonb_set $($ data, ' $\{$ friends, 999$\}$ ', "'Andrea"', true);

To replace the particular element of an array, the array name and the position of the element have to be specified inside the array. (The first element of an array is indexed by 0 .)

Example 12

-- Replace the $\mathrm{n}$-th element of an array with the given name

UPDATE json_table

SET data = jsonb_set(data, '\{friends, 1$\}$ ', '"Leo"');

PostgreSQL does not actually update a value of the JSON data. It creates a temporary version of the data, which is modified according to the particular statement. After that the modified version is assigned back to the column.

Additionally, PostgreSQL allows the specification of a sequence of modifications inside one statement, as shown in the following example. That way, the requirement in the framework that the system supports a full update operation is fulfilled.

Example 13

UPDATE json_table

SET data $=$ jsonb_set (

jsonb_set(data \#- '\{friends,-1\}', '\{friends,999\}', '"Andrea"', true), '\{name\}', '"Pete"');

\subsection{MySQL}

Starting with version 5.7.8, MySQL supports JSON update operations. The main property of MySQL/JSON is support of the native JSON data type defined by RFC 7159 as the only RDBM system discussed in this paper. This data type provides efficient access to data stored in JSON documents over storing them as strings in string columns.

Example 14

-- Create a table with the column which contains a JSON

-- document 
CREATE TABLE t2 (jdoc JSON);

-- Insert a row

\section{INSERT INTO t2}

VALUES('\{ "who": "Fred" ,"where": "BBC",

"friends":[\{"name": "Lili", "rank":5\},\{"name": "Hank", "rank": $7\}]\} ')$;

Example 15

-- Inserts a new object with the key/value pair:

-- "location": "Philadelphia"

SELECT JSON_INSERT (jdoc, '\$.location', 'Philadelphia')

\section{FROM t2;}

The requirements of the framework ("Insert an array member before/after another array member") is not supported. The JSON_ARRAY_INSERT function allows inserting into an array within the document specifying its location.

Example 16

-- Inserts the object: \{"name": "Joe", "rank":5\}

-- as the second object of the array

SELECT JSON_ARRAY_INSERT (jdoc, '\$.friends[1]', '\{"name": "Joe", "rank":5\}')

\section{FROM t2;}

MySQL offers the JSON_ARRAY_APPEND function, which appends the specified element at the end of the named array.

Example 17

SELECT JSON_ARRAY_APPEND (jdoc, '\$.friends', '\{ "name": "Mary", "rank":5\}')

FROM t2;

The following two examples show how the JSON_REMOVE function can be used to delete an object and the n-th array element, respectively. (The second example deletes the second element of the array called friends.)

\section{Example 18}

-- 2.1Delete an object with the given name

SELECT JSON_REMOVE (jdoc, '\$.where')

FROM t2;

Example 19

-- Delete the second element of the array

SELECT JSON_REMOVE (jdoc, '\$.friends[1]')

FROM t2;

\begin{tabular}{|c|c|c|c|}
\hline UPDATE-Operations & SQL Server & PostgreSQL & MySQL \\
\hline a) & INSERT & + & + \\
Insert an object & & $-/+$ & + \\
bey/value) & Insert an array \\
element before another one & & & \\
c) & & & \\
\hline
\end{tabular}

The following two examples show how the JSON_REPLACE function can be used to replace the value of the specified name/value pair and the n-th array element, respectively. The second example replaces the value of object called "rank" of the second array element.

\section{Example 20}

-- 3.1 Replace the value of the object with the given name

SELECT JSON_REPLACE (jdoc, '\$.where', 'CNN') FROM t2;

Example 21

-- 3.2 Replace the $n$-th element of the array

SELECT JSON_REPLACE (jdoc, '\$.friends[0].rank', 8) FROM t2;

MySQL supports also the JSON_SET function, which inserts or updates data in a JSON document. This function is semantically identical to the SQL MERGE statement: The former upserts the content of a JSON document, while the latter upserts the content of a table.

Example 22

-- Replaces the value and inserts the new object

SELECT JSON_SET (jdoc, '\$.where', 'ABC',

'\$.gender', 'M') FROM t2;

\section{SUMMARY}

We started this paper by listing all update primitive operations for JSON documents.

Also, the existing implementations in RDBMSs for JSON update have been examined. SQL Server uniformly supports all implemented operations with the JSON_MODIFY function, but the syntax of the second argument of the function is not uniform. Also, the use of the NULL value to delete an object with the given key is difficult to understand. SQL Server supports the full update operation using the invocation of the SQL_MODIFY function inside another SQL_MODIFY.

The syntax for PostgreSQL update operations is rather awkward and there is no any uniformity for update primitives concerning objects and array elements of a JSON document. On the other hand, PostgreSQL supports the full update operation in the same way as this paper proposes it, using the comma sign to combine several operations.

MySQL has the full support for our framework. The only difference in MySQL implementation is the art of ordering inside JSON arrays. The JSON_ARRAY_INSERT function allows inserting into an array within the document specifying its location (first, second, third, etc.) and not its position in relation to the existing array elements, as it is given in the framework.

The table given above shows the support of update primitives specified in the three DBMSs. 


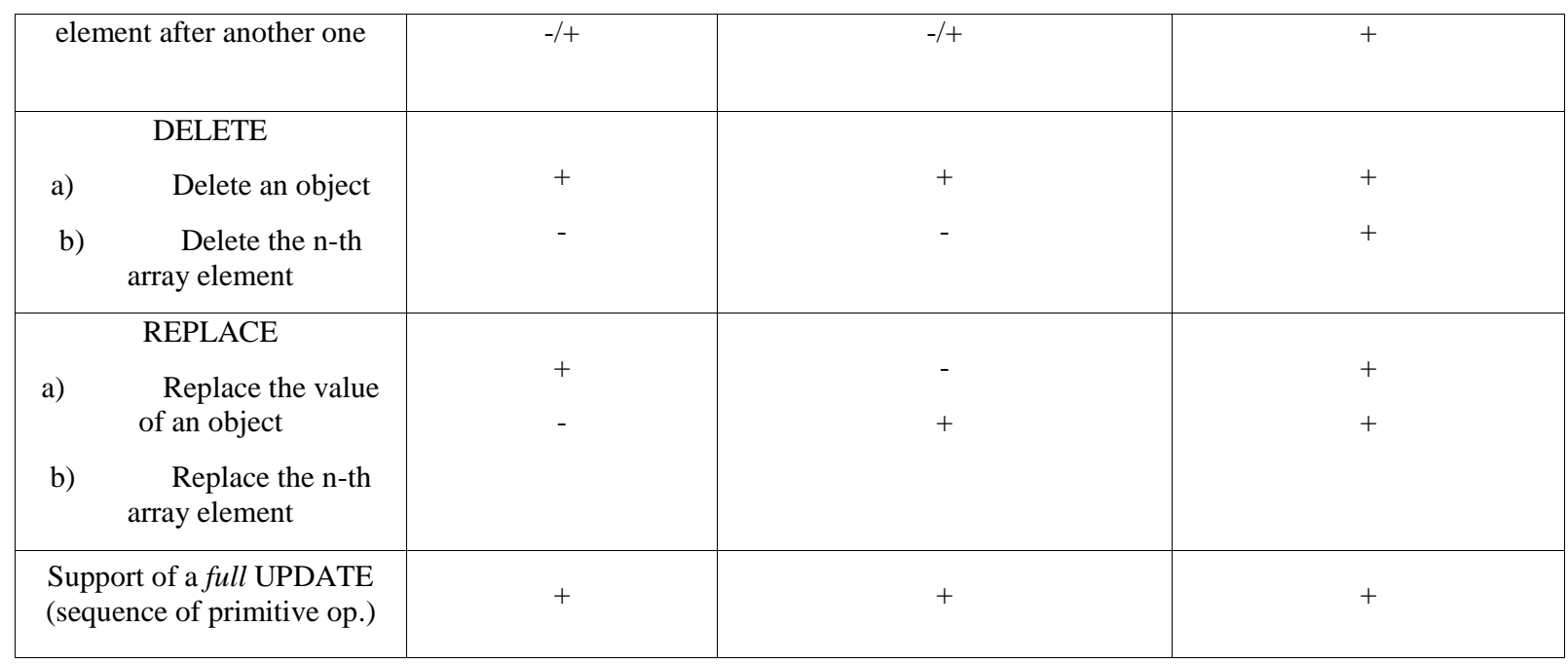

\section{REFERENCES}

[1] Chausser, C. 2013. Enabling JSON Document Stores in Relational Systems. WebDB.

[2] ISO/IEC TR 19075-6:2017. Information technology -Database languages -- SQL Technical Report -- Part 6: SQL support for JavaScript Object Notation (JSON). http://standards.iso.org/ittf/PubliclyAvailableStandards/ind ex.html. . (Accessed October 15, 2019)

[3] Liu, Z,H, Hammerschmidt, B., McMahon, D. 2014. JSON Data management - Supporting Schema-Less Development in RDBMS. SIGMOD/PODS'14.

[4] Liu, Z.H. 2019. JSON Data Management in RDBMS. In The Emerging Technologies and Applications in Data Processing and Management.

[5] Liu, Z.H, Gawlick, D. 2015. Management of Flexible Schema Data in RDBMSs. CIDR' 15.

[6] MS SQL Server: JSON Data, https://docs.microsoft.com/en-us/sql/relationaldatabases/json/json-data-sql-server. (Accessed Dec 25, 2019)

[7] MS SQL Server JSON_MODIFY function. https://docs.microsoft.com/en-us/sq1/t-sql/functions/jsonmodify-transact-sql?view=sql-server-2017. (Accessed July $5,2019)$

[8] Petković, D. 2017. SQL/JSON Standard: Properties and Deficiencies. Datenbank Spektrum, Vol. 17, No.3.
[9] Petković, D. 2017. JSON integration in relational database systems. Int. Journal of Computing Applications, Vol. $168(5)$

[10] Petković, D. 2018. Full-Text Search Extensions for JSON Documents: Design Goals and Implementations. In Proc. Of the 14th International Conference, BDAS 2018, Poznan, Poland.

[11] PostgreSQL9.5,JSONTypes,www.postgresql.org/docs/9.5/ static/datatype-json.html (accessed November 13, 2019)

[12] PostgreSQL 9.5, JSON Functions www.postgresql.org/docs/9.5/static/functions-json.html (Accessed December 20, 2019)

[13] Tatarinov, I.; Ives, Z.; Halevy, A.; Weld, D. 2001. Updating XML. ACM SIGMOD.

[14] Zemke F, Hammerschmidt B, Kulkarni K, Liu Z, McMahon D, Melton J, Michels J, Özcan F, Pirahesh H. ANSI SQL/JSON: Part www.wiscorp.com/pub/DM32.2-2014-00025r1-sql-jsonpart-1.pdf. (Accessed July 26, 2019).

[15] Zemke, F.; Hammerschmidt, B. ; Kulkarni, K. ; Liu, Z. ; McMahon, D. ; Melton, J. ; Michels, J. ; Özcan, F. ; Pirahesh, H. - ANSI SQL/JSON: Part 2 : Querying JSON, www.wiscorp.com/pub/DM32.2-2014-00025r1-sql-jsonpart-2.pdf. (Accessed October 25, 2019) 